\title{
Visual Analysis of Charge Flow Networks for Complex Morphologies
}

Sathish Kottravel, Martin Falk, Talha Bin Masood, Mathieu Linares and Ingrid Hotz

The self-archived postprint version of this journal article is available at Linköping University Institutional Repository (DiVA):

http://urn.kb.se/resolve?urn=urn:nbn:se:liu:diva-160177

N.B.: When citing this work, cite the original publication.

Kottravel, S., Falk, M., Masood, T. B., Linares, M., Hotz, I., (2019), Visual Analysis of Charge Flow Networks for Complex Morphologies, Computer graphics forum (Print), 38(3), 479-489.

https://doi.org/10.1111/cgf.13704

Original publication available at:

https://doi.org/10.1111/cgf.13704

Copyright: Wiley (12 months)

http://eu.wiley.com/WileyCDA/ 


\title{
Visual Analysis of Charge Flow Networks for Complex Morphologies
}

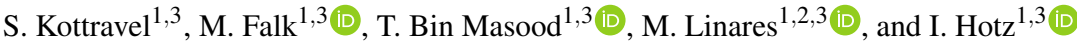 \\ ${ }^{1}$ Scientific Visualization Group, Linköpings University, Sweden \\ ${ }^{2}$ Laboratory of Organic Electronics, Linköpings University, Sweden \\ ${ }^{3}$ Swedish e-Science Research Centre (SeRC)
}

\begin{abstract}
In the field of organic electronics, understanding complex material morphologies and their role in efficient charge transport in solar cells is extremely important. Related processes are studied using the Ising model and Kinetic Monte Carlo simulations resulting in large ensembles of stochastic trajectories. Naive visualization of these trajectories, individually or as a whole, does not lead to new knowledge discovery through exploration. In this paper, we present novel visualization and exploration methods to analyze this complex dynamic data, which provide succinct and meaningful abstractions leading to scientific insights. We propose a morphology abstraction yielding a network composed of material pockets and the interfaces, which serves as backbone for the visualization of the charge diffusion. The trajectory network is created using a novel way of implicitly attracting the trajectories to the skeleton of the morphology relying on a relaxation process. Each individual trajectory is then represented as a connected sequence of nodes in the skeleton. The final network summarizes all of these sequences in a single aggregated network. We apply our method to three different morphologies and demonstrate its suitability for exploring this kind of data.
\end{abstract}

CCS Concepts

- Human-centered computing $\rightarrow$ Scientific visualization; • Computing methodologies $\rightarrow$ Discrete-event simulation; • Applied computing $\rightarrow$ Physical sciences and engineering;

\section{Introduction}

The development of organic solar cells plays an increasingly important role as they have a great potential as an alternative energy source. They offer many benefits compared to conventional solar cells since they can be manufactured in high throughput at low prices. The major challenge with organic solar cells is their limited efficiency, which cannot yet compete with conventional solar cells. In a nutshell, an organic solar cell consists of two different materials that serve as donor and acceptor for charges. Photon absorption in the material leads to the formation of an exciton, which migrates to the interface to be separated in a hole and an electron, and then transported toward the electrodes. Thereby, the donor material transports the holes and the acceptor material the electrons. Figure 1a illustrates the process for one charge pair. The number of generated charge pairs and their success in reaching the electrodes without recombination is responsible for the efficiency of the solar cell and is largely controlled by the morphology of the material. Besides the obvious relevance of the morphology, there are many other effects influencing the performance of the material. These are, among others, charge interactions, temperature, external electrical fields and polarization effects in organic materials through atomic induced dipoles on nearby molecules [VKN*16]. Therefore, it is important to consider the actual transport of the charges within

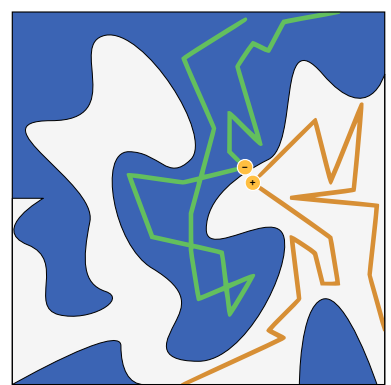

(a)

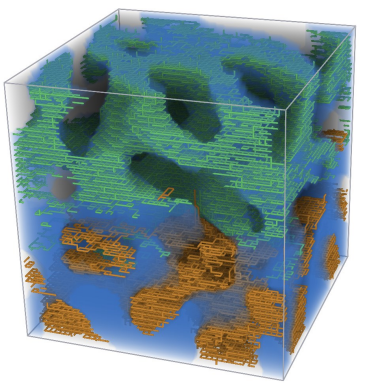

(b)
Figure 1: (a) Setup of an organic solar cell consisting of two interpenetrated materials. (b) Direct visualization of the trajectories obtained from a Kinetic Monte Carlo simulation. Electron trajectories are displayed as green lines, hole trajectories as orange lines. The acceptor material (blue) provides the morphological context.

the material and it is not sufficient to only analyze the morphology. Kinetic Monte Carlo (KMC) simulations are widely used to study charge transport in organic materials since they provide a fast 


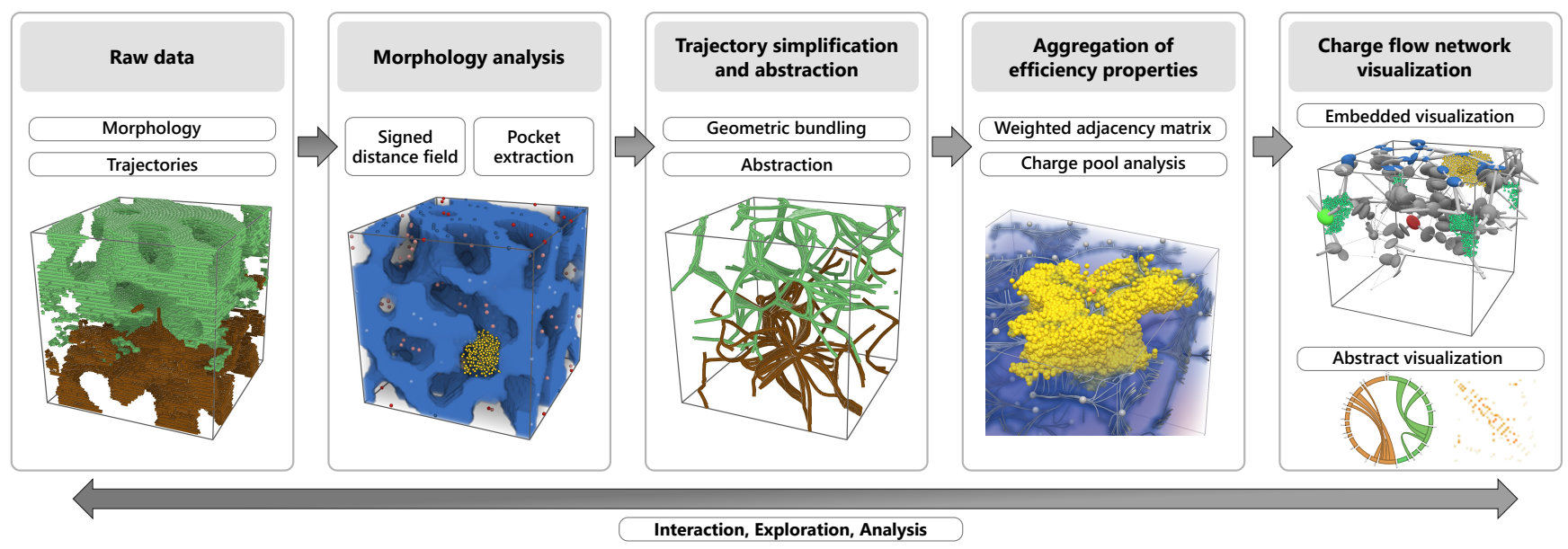

Figure 2: Overview of our charge flow network pipeline. Given the raw data, the pipeline generates the network abstraction and network visualization.

way to explore realistically the complex morphologies going further than the simple shorter paths. Using KMC simulations it is possible to represent the quantum mechanical interactions in the material responsible for the charge transport [JLS12, VSL15]. The results of these simulations are ensembles of pairs of charge trajectories, which represent possible realizations of the charge transport, see Figure $1 \mathrm{~b}$ for a direct visualization. Understanding the differences or similarities between the geometric properties of the morphology and the actual paths of the charges is one of the major questions behind this research.

In this paper, we present a visualization system resulting from a long collaboration between domain scientists and visualization experts. At the beginning of the project, neither side was clear about what is needed or what is feasible. Thus, the first visualization framework was built on standard visualization concepts. It combines various diagram views of scalar efficiency measures with linked 3D views for interactive filtering and exploration [KVL* 17]. This framework for the first time gave the domain scientists the possibility to explore their data visually. However, using the system, soon new demands especially for multi-scale exploration on different abstraction levels emerged.

The result is the construction of a 'charge flow network'. The network forms an abstraction of the trajectories embedded in the morphology. In this setting, the morphology is represented as a set of sub-domains of material morphology and layer of interface between the sub-domains. From here forth, for simplicity we call them as pockets and pocket interfaces. Each trajectory is described as a sequence of visited material pockets and the associated dwell times. The sum of all trajectories constitutes the charge flow network, which is augmented with aggregated characteristic properties of the original trajectories. The system supports the selection of pockets, interfaces, and trajectories and facilitates a detailed analysis of the charge transport through user interaction on all levels. Hole and electron pairs can be followed through the network thereby identifying possible transport bottlenecks, which can be re- lated to the pocket interface geometry or charge interaction on the morphology surface.

Within this paper, we make the following contributions:

- Introduction of the concept of transport networks as an abstraction for movements in complex morphologies.

- Novel approach for line bundling in constrained morphologies.

- Aggregation of novel statistical measures for charge distributions in morphological pockets.

- An interactive visualization system for the visual analysis of charge transport networks across different abstraction levels.

The paper is structured as follows. Section 2 gives details about the data and the application requirements and how those are approached by the system. After the related work Section 3, the extraction of the network is described in Section 4. The visual exploration of these networks in discussed in Section 5. We guide through a couple of use cases in Section 7. Finally, the paper concludes in Section 8.

\section{Domain specific goals and basic concept}

Background and requirements. Though the performance of organic devices is improving, a full theoretical understanding of the physics of organic materials is still missing. Their properties differ significantly from crystals and the charge carriers tend to localize in preferred regions in the morphology. Theoretical investigations of the charge mobility and transport are based on Kinetic Monte Carlo (KMC) simulations [VKN*16]. The underlying physical model is evolving continuously and new physical interactions are integrated to make it more realistic.

The domain specific goals are two fold; at first, to extend quantum mechanical models to realistically describe the transport of charges in organic material. The second goal for these specific calculations is to understand the relation between the geometric properties of the morphology and the charge transport. Specifically, this is the distribution of the charges within the morphology, the probability of charge recombination at the morphology boundary and the 
identification of the bottlenecks that are not purely related to the morphological geometry. Correspondingly the visualization tasks are related to model verification and charge flow exploration.

Model verification requires the evaluation of basic macroscopic transport properties. Typical questions to be answered are how the charges progress toward the electrodes and how quickly they reach them. Therefore, an abstraction from the stochastic movements and a simplified charge path representation is essential. For an efficiency analysis, the identification of bottlenecks and the risk for charge recombination are of special interest. Bottlenecks can be related to large retention times of charges in certain preferred regions. An important indicator for the risk of recombination of electrons and holes is their distance to the material interface.

The concept of the charge flow network. An important aspect of charge transport simulations is to understand how charges move within a given morphology. The charge movement does not only depend on the geometry of the morphology but also on the electrostatic potential and the small-scale quantum mechanic configuration. This information is contained within the trajectories. Therefore, it is essential to extract the connectivity information from the trajectories.

The charge flow network proposed in this paper is designed to serve these requirements by providing an easy way to follow the large scale transport of the charges in the morphology. Preferred locations for the charges can be associated with morphology pockets and bottlenecks can be identified by the analysis of interfaces. The network provides a valuable means to aggregate statistical information necessary for the recombination analysis from the pockets and their interfaces.

Algorithmic pipeline. Our proposed framework embeds the charge transport and its properties in an interactive flow network (see Figure 2). The morphology and the ensemble of trajectories serve as the input of the pipeline. A topological morphology analysis defines the pockets, which subsequently serve as nodes for the abstraction and simplification of the trajectories. The last step in the pipeline is the visualization of the result either as an abstract 2D graph or a spatial network embedded in the morphology. Characteristic measures of the pockets and the interfaces can be inspected in both representations. The individual steps of the pipeline will be discussed in Section 4.

Data generation. The data consists of ensembles of charge trajectories embedded in a material morphology. The morphologies are generated using the Ising model for two materials with a volume ratio of 50:50 resulting in morphologies with different interpenetration. They are represented as a binary volume consisting of donor sites represented by ' 1 ' and acceptor sites represented by ' 0 '. For this study, we have saved two morphologies with different interpenetration levels as indicated by different area-volume ratios. The dimensions of the boxes obtained is $18 \times 18 \times 28$.

The motion of the charges in the morphology is followed using a KMC procedure based on the Marcus equation. At the beginning the charges (electrons and holes) are placed at the interface between the two materials (donor and acceptor, respectively). An electric field is applied in the $Z$ direction of the box inducing the splitting of the charges and the motion toward the electrodes. For each morphology, we have performed at least $200 \mathrm{KMC}$ simulations and saved the trajectories for both charge carriers, namely holes and electrons.

Each trajectory is assembled from a discrete series of transitions between the sites representing a stochastic movement through the material. They are represented as a list of site IDs augmented with a certain dwell time at the site. The simulation assumes periodic boundary conditions in the horizontal plane. The Gromacs file format [LHvdS01] is used for the morphology and KMC simulations are performed in Gorilla [VKN*16].

\section{Related work}

Aspects of our work can be found in different fields of visualization: abstraction of complex morphologies through networks; rendering large sets of trajectories; and ensemble data. Each of these aspects bears some similarities to our work, which we will summarize briefly in this section.

In several applications, the extremal structure of the distance field of porous materials has been used to build a graph structure to represent the material morphology [GRWH12]. Shivashankar et al. [SPN*16] use similar abstractions to find filament structures within density fields from the cosmology domain. Aboulhassan et al. [ABW*15] focus on the exploration of the backbone of the morphological structures of organic solar cells to detect geometric bottlenecks. This backbone has some similarities to parts of our network construction however without the consideration of the trajectory data. This work has been extended by proposing a comparative visual analysis framework for parameter space exploration for local morphology features [ASB*17]. The framework is built on shape-based clustering of local cubical regions of the morphology that they call patches.

Ushizima et al. [UMW*12] use an augmented pore network for the analysis of $\mathrm{CO}_{2}$ storage in rock formations. Another application using a similar abstraction concept has been presented by Aldrich et al. [AHK*17]. They construct a discrete fracture network to characterize the flow in constrained networks in geoscience applications. An exploration framework based on such methods using multiple views, filtering, brushing and linking for charge flow data was recently presented by Kottravel et al. [KVL*17]. Hierarchical edge bundling introduced by Holten [Hol06] has been used in many applications dealing with large sets of lines.

The analysis of trajectories with similar characteristics plays also a role for motion tracking and movement data. Though the applications are different, they both deal with non-smooth trajectories allowing crossings. Andrienko et al. [AA12] presented a task classification with four categories: looking at trajectories, looking inside trajectories, bird's-eye view on movement, and investigating movement in context. All of these are of importance in our application. An essential difference, however, is the dimensionality; the $3 \mathrm{D}$ charge trajectories cannot easily be projected onto $2 \mathrm{D}$ maps. Also the kind of interactions between trajectories is fundamentally different and thus most methods are not transferable. 


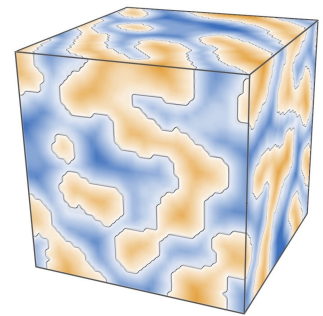

(a)

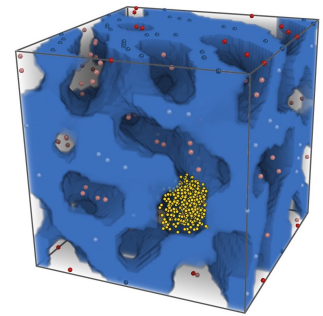

(b)

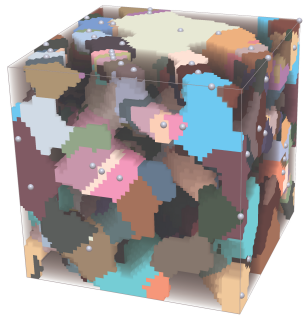

(c)

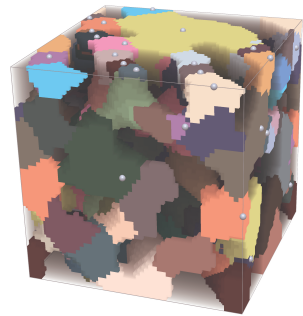

(d)

Figure 3: Pocket extraction. (a) The distance field computed for the morphology (acceptor material in blue, donor material in orange). (b) Red and blue points show the maxima of the distance field, while yellow points show the voxels in the ascending manifold of one of the maxima, i.e. one pocket. (c) morphology segmentation corresponding to 182 originally extracted local maxima. (d) the segmentation corresponding to 125 pockets obtained after pocket simplification.

\section{Charge flow network extraction}

The charge flow network is the key abstraction around, which the application is built. It is constituted from pockets and connecting interfaces. While the pocket definition is based on the morphology, the connectivity is established on the basis of the trajectories. The individual components will be described in the following sections.

\subsection{Morphology analysis}

Typically a morphological structure is analyzed by means of its topological skeleton. However, a one dimensional skeleton is not sufficient to represent the distribution of the charges in the morphology. Therefore, we are more interested in morphology pockets, which are later used as anchor points for the trajectory simplification.

Pocket extraction. The morphology pockets are represented by the maxima of the signed distance field of the morphology, see Figure 3a. The distance transform is calculated using the algorithm proposed by Saito et al. [ST94] using an Euclidean metric. The local maxima are extracted by a simple voxel-based neighborhood analysis; we consider a 26-voxel neighborhood. To avoid artifacts due to plateaus, we follow the concept of symbolic perturbation using the index of the voxels as introduced by Edelsbrunner et al. [EM90]. The result is a list of pocket representatives carrying a unique index, indicated by red and blue points in Figure $3 \mathrm{~b}$. The pockets are defined as the set of voxels in the ascending manifold of the maxima, depicted as yellow points in Figure $3 \mathrm{~b}$.

Since the top and bottom layers of the material play a special role, we distinguish between material pockets and surface pockets. Surface pockets are pockets that have a direct connection with the electrodes. They are represented by maxima of the twodimensional distance field of the surface layers, which ensures that the simplified trajectories are always connected to the electrodes.

Pocket simplification. A typical approach to simplify topological structures is the use of persistence [EH08]. Persistence is a measure that does not take the geometric embedding of the structure into account. Therefor we introduce a different strategy that considers the geometry and the the value of the distance field in the maxima.

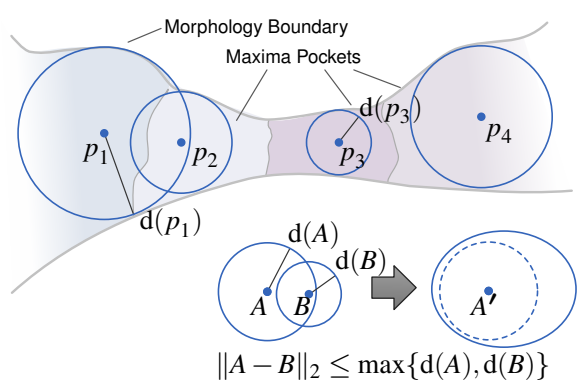

Figure 4: A pair of pockets is merged if either center is located within the influence sphere of the other pocket.

To remove small pockets that are closely attached to a larger pocket we introduce a pocket merging step following a simple rule: a pair of pockets is merged if either center is located within the influence sphere of the other pocket. For pockets $A$ and $B$ with distance values $\mathrm{d}(A)$ and $\mathrm{d}(B)$, this means

$$
\|A-B\|_{2} \leq \max \{\mathrm{d}(A), \mathrm{d}(B)\}
$$

The merging process starts from the largest pocket and is applied as long as an adjacent pocket with smaller radius satisfying condition 1 is available. The process continues from the next largest pocket until no more merges are possible (see Figure 4). This guarantees that no pockets with holes, e.g. loops, are generated. The pocket is represented by the location of the largest maxima. The resulting volume segmentations are shown in Figures $3 \mathrm{c}$ and $3 \mathrm{~d}$.

\subsection{Trajectory abstraction}

The connectivity of the network is established by the trajectories that are individually represented as a sequence of pockets or a 'word' where the pocket indices serve as the alphabet. The raw words contain large sequences of constant letters when a charge remains in one pocket, and sequences of alternating letters when a charge transfers between pockets. To reduce a word to its essential pockets and transitions a set of simple grammar rules is applied.

Rule 1: Establishing stable transitions. Charges often jump back and forth between interface voxels of neighboring pockets until 
they finally stay in the new pocket. Therefore, we ignore all transitions until the charge stays for a minimum amount of jumps minlen within the new pocket.

$$
x \underbrace{y \ldots y}_{\leq \text {minlen }} x \rightarrow x x
$$

For example, consider the word 'aaaabbaaaccc.' Rule 1 removes pocket ' $b$ ' if minlen $>2$ thus yielding the word 'aaaaaaaccc.' However, a transition pocket ' $c$ ' in word 'aaaacddd' will not be removed.

Rule 2: Representing each visited pocket by one letter. Consecutive entries of the same letter are collapsed into a single letter, e.g. 'aaaaacddd' will yield the final word 'acd.'

$$
x x \rightarrow x
$$

For each trajectory word, we create an adjacency matrix summarizing all transitions of the trajectory. To keep track of the frequency of transitions between the pockets each matrix entry $x_{i, j}$ represents the total number of transitions from the letters $x_{i}$ to $x_{j}$ and, thus, the connectedness of pockets. The diagonal entries are used to represent the number of charges contributing to one pocket. For the complete network, all matrices are assembled by adding the nondiagonal entries and keeping the diagonal elements. The composed adjacency matrix provides all relevant information to create a directed graph of the morphology. The trajectory words and matrices provide an efficient and compact representation of the trajectories and are later used as the basis for their exploration.

\subsection{Trajectory based morphology skeleton}

While the charge flow network summarizes the charge path in an efficient way, the spatial embedding of this path is no longer represented. To compute exact geometric measures we require the morphology skeleton as a reference.

To obtain the skeleton, we perform a constrained trajectory bundling, which straightens the trajectories while also pulling them toward the center of the morphology. The morphology's center is implicitly defined by the corresponding distance field (cf. Figure 3a).

The bundling is performed by employing a spring mass system in combination with an external force defined by the gradient of the distance field. Thus, charges are attracted by the ridges of the morphology. A line of springs connects all charge positions within each trajectory. Note that each trajectory corresponds to one independent spring chain. To obtain a more even distribution of the charges along the relaxed trajectories we modify the linear behavior of the springs (Hook's law) by adding a cubic term. Thereby ensuring that no topological feature of the morphology is lost. The Verlet integration scheme is used to solve the system.

For our datasets, 200 to 500 steps were sufficient for relaxing the entire system into the skeleton. The external forces pull them at first toward the extremal surfaces and then to the extremal lines of the distance field, see Figure 5. Planar structures already become prominent after about 50 iterations and are be used as reference for the geometric measures.

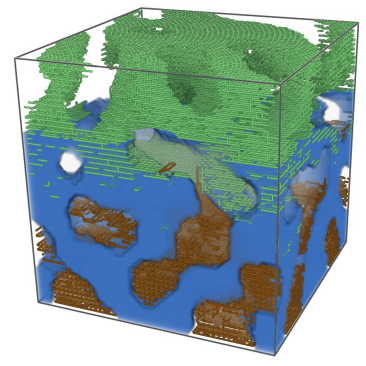

(a) Initial positions

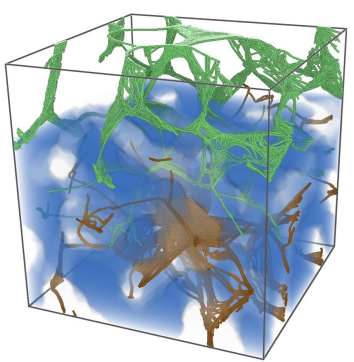

(c) 80 iterations

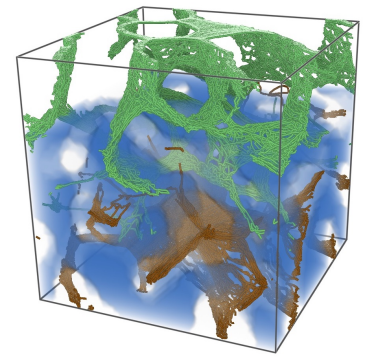

(b) 40 iterations

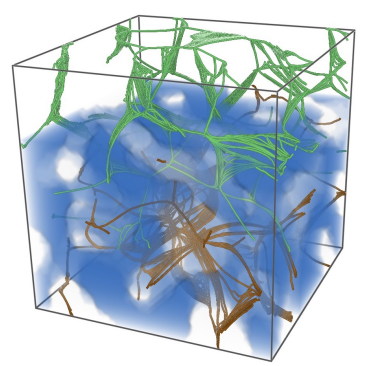

(d) 200 iterations
Figure 5: Trajectory bundling using a spring mass system embedded in a force field, which is defined by the gradient of the distance field, During the bundling process the trajectories are first drawn to planar structures before they move toward the ridges of the morphological skeleton.

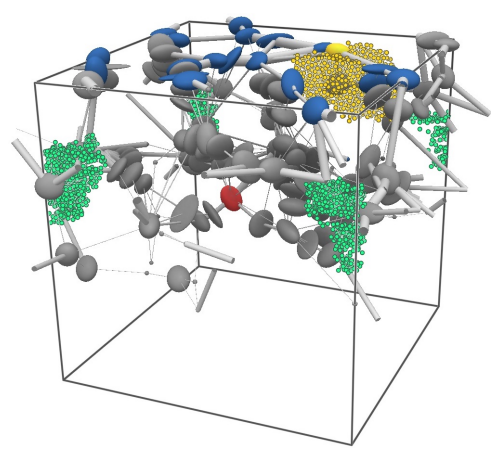

(a)

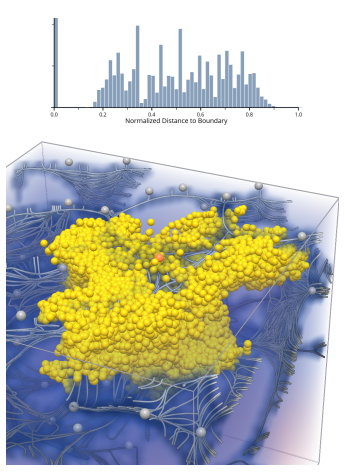

(b)
Figure 6: (a) The charge flow network for one material of the morphology. The starting pocket is highlighted in red while the end pockets are shown in blue. Two selected pockets and the associated charge positions are shown in green and yellow, respectively. Note that the periodic boundary conditions are taken into account indicated by the open-ended connections. (b) Top: Radial density plot for the yellow pocket shown below. Bottom: The charge positions within a pocket of interest are highlighted in yellow. The morphology skeleton represented as lines and the volume rendering of the distance field of the skeleton are shown for context. 


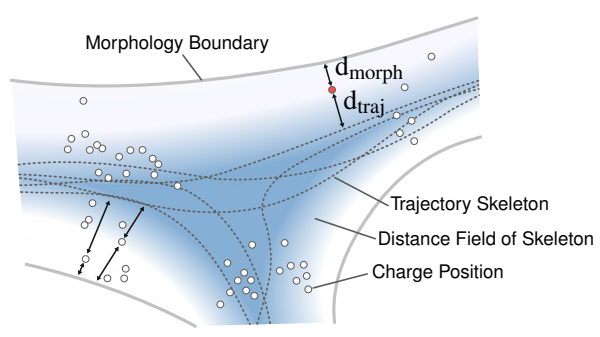

Figure 7: Radial pocket charge distributions. The radial distance $r$ for a given charge position (red) depends on the distance to the morphology boundary $\mathrm{d}_{\text {morph }}$ and the distance to the skeleton $\mathrm{d}_{\text {traj }}$.

\section{Network efficiency measures}

The network provides the basis to aggregate geometric efficiency indicators for the charge transport as the charge distribution in pockets and the transitions between the pockets.

\subsection{Pocket exploration}

Pockets are a connected component resulting from the morphology segmentation. They are represented by the largest local maximum within the pocket. In the abstract network, they are displayed as ellipsoids representing either the geometrical shape of the pocket or the distribution of the associated charges. The ellipsoid results from a principal component analysis of the pocket voxels or the charge distribution, respectively, see Figure 6a. Different properties can be mapped to the color of the ellipsoid glyph. Typical examples are start or end pockets, number of charges, and pocket size. Other provided mappings include average charge density and the net flow through the pocket. All these parameters can alternatively be mapped to the size of the ellipsoid. All charge positions associated with a pocket can be highlighted (Figure 6b).

Pocket classification. A typical classification of geometric shapes that we use for the pockets employs the anisotropy measures: spherical anisotropy $c_{s}$, planarity $c_{p}$, and linearity $c_{l}$. The measures are defined in terms of the eigenvalues $\lambda_{i}, i=1,2,3$ of the charge distributions in the pockets as $c_{s}=\frac{3 \lambda_{3}}{\lambda_{1}+\lambda_{2}+\lambda_{3}}, c_{p}=\frac{2\left(\lambda_{2}-\lambda_{3}\right)}{\lambda_{1}+\lambda_{2}+\lambda_{3}}$, and $c_{l}=$ $\frac{\lambda_{1}-\lambda_{2}}{\lambda_{1}+\lambda_{2}+\lambda_{3}}$. Every pocket is represented as a point in the barycentric space spanned by $c_{s}, c_{p}$, and $c_{l}$, see Figure 11 , bottom.

Pocket charge distributions. An important characteristics of the pockets is the distribution of enclosed charges and their proximity to the material interface. Charges close to the boundary exhibit a high chance for charge recombination. We define a relative proximity measure with a value of 1 on the material interface and a value of 0 at the morphology skeleton represented by the trajectory bundles. It is computed from the shortest distance to the morphology boundary $\mathrm{d}_{\text {morph }}$, given by the distance field of the morphology and the distance to the skeleton $d_{\text {traj }}$. The concept is illustrated in Figure 7. To obtain the value $\mathrm{d}_{\text {traj }}$, we compute an additional distance field with respect to the extracted skeleton. Since pockets exhibit different sizes and shapes the value is normalized. For a given

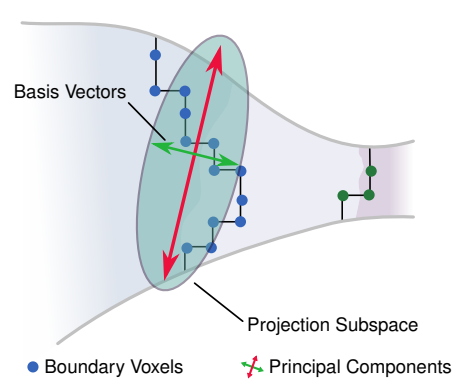

Figure 8: Planar pocket interfaces are constructed using principal component analysis of the boundary voxels.

charge position $p$, the normalized radial distance $r$ is defined as

$$
r=\frac{\mathrm{d}_{\text {traj }}(p)}{\mathrm{d}_{\text {morph }}(p)+\mathrm{d}_{\text {traj }}(p)}
$$

The radial density plot of a single pocket is obtained by computing the histogram over the radial distances of all charges located inside this pocket (Figure $6 \mathrm{~b}$ ).

\subsection{Pocket interface exploration}

For the investigation of transport bottlenecks, the pocket interfaces are of special interest. This comprises their cross-section geometry and the distribution of the locations of the charges when passing from one pocket to the next. Therefore, we extract the pocket interfaces as explicit geometry and use them to display some transport characteristics.

Extracting the interface geometry between a pocket pair involves three steps, which are illustrated in Figure 8. At first, interface voxels are identified as voxels having different pockets in their $3 \times 3 \times 3$ neighborhood. The interface surface is obtained by means of a principal component analysis of the interface voxels. The two largest principal components span a plane positioned at the center of mass of the interface voxels. The 2D convex hull of the projected interface voxels determines the extent.

We augment these cross sections with a heatmap showing the distribution of the charge transitions where we distinguish between inflow and outflow. The heatmap of the outflow is generated by scanning each trajectory for transitions from pocket $A$ to $B$. All intersection points are subsequently splatted onto the interface surface using Gaussian splats. By considering transitions from $B$ to $A$ we can obtain the inflow of pocket $A$. Figure 9 shows the pocket interfaces computed for a single pocket. Each interface is rendered in-place at the corresponding cross section of the morphology. The heatmaps on these interfaces display the charge flow in blue.

\section{Visualization and interaction}

To visualize the data, we provide three different options: geometrically embedded trajectory bundles, abstract charge flow networks embedded in $3 \mathrm{D}$, and $2 \mathrm{D}$ chord diagrams. All visualizations are based on the three data structures we have defined above: the morphology pockets, the adjacency matrices for the trajectories, and 

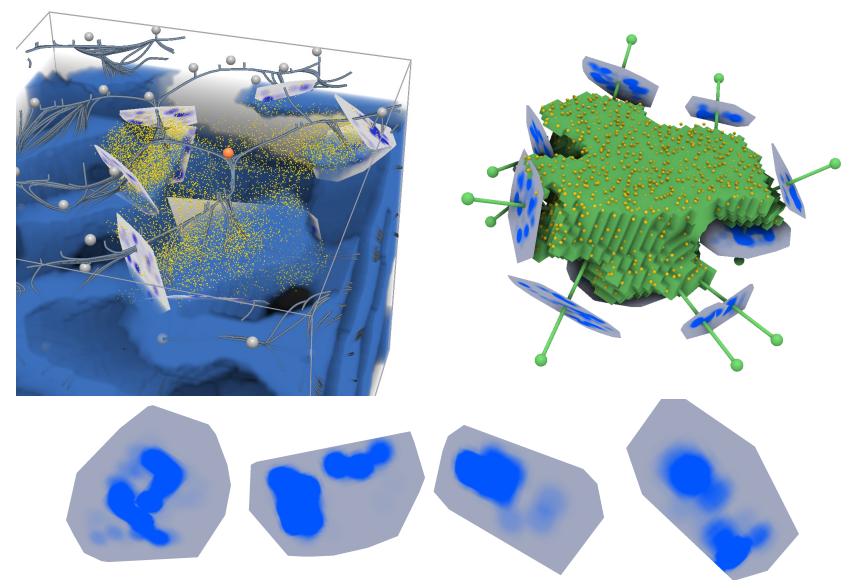

Figure 9: Pocket interfaces between a single pocket (orange) and all its neighbors. Charge transitions from one pocket to another are represented as heatmap on the cross section: inflows from neighboring pockets (top left) and corresponding outflows (top right) embedded in the network visualization; extracted cross sectional pocket interface hulls (bottom). Charges (yellow) are only shown for the selected pocket.

the bundles obtained by the relaxation. Different rendering styles using volume rendering and explicit geometry are provided by the system. All parts of the network and the geometry can be interactively selected and separately clipped or highlighted. The different representations are all linked to each other and can be interactively explored. In all representations, pockets or trajectories can be selected. Alternatively one can iterate through the pockets or trajectories using the keyboard. The presented concepts are implemented in C++ and OpenGL using the Inviwo framework [JSS*19]. $\mathrm{D} 3$ [BOH11] is being used for the generating the chord diagrams.

Morphology representation. For the 3D visualizations, the morphology can be rendered as contextual information either as isosurface or as semitransparent volume. The segmentation process can be evaluated by showing the pockets either by using their centers, the contributing charges or the volume. Pockets and their connectivity can also be inspected individually as shown in Figure 9. On demand pocket interfaces can be highlighted. All scalar measures can integrated into the volume, for example the distance field or the charge density.

Trajectory bundle representation. In this representation, all levels of the trajectory relaxation can be shown from the raw trajectories up to the skeleton. Either individual trajectories or the entire ensemble can be visualized while pocket centers and/or charge positions can be added on demand. The morphology can be rendered as context.

Charge flow network. Embedded in the three dimensional volume, the charge flow network shows the pockets at their actual geometric position as ellipsoids. The connections between the pockets are displayed as cylindrical connectors whose size can be for

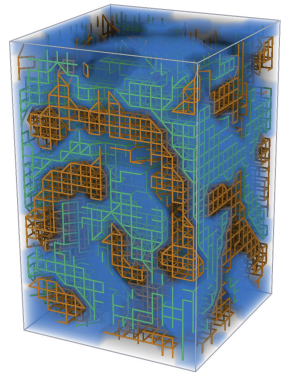

(a) Raw DATASET2

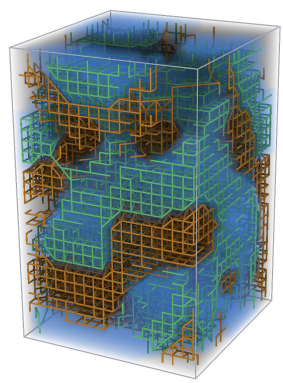

(c) Raw DATASET3

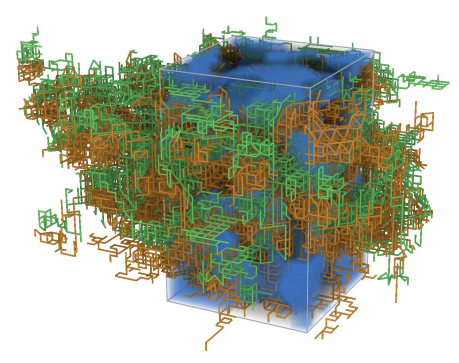

(b) Expanded trajectories DATASET2
Figure 10: Two datasets in comparison. Left: trajectories embedded within the morphology. Right: expansion of the trajectories considering periodic boundary conditions.

example mapped to the flow between the pockets. Figure 6 a illustrates the result for the abstraction of all trajectories in one material and gives an overview of the network and the connectivity between nodes. The pocket volumes and the charge distributions for selected pockets can be added on demand.

2D chord diagram. A different, more abstract variant of the charge flow network is the chord diagram [KSB*09] which does not include any spatial information. The chord links indicate direct relationships between two pockets where the thickness of each link close to its start reflects the number of transitions from the source to the target. This representation allows to get a qualitative overview of all pocket size, their valency, and contribution to the charge transport. As such they are a valuable interface to select trajectories or pockets for further inspection.

\section{Use cases}

We explore the charge propagation in three different datasets using the framework introduced. DATASET 1 is a relatively large dataset that has been used for all illustrating purposes above. The other two datasets have the same volumetric extent (dimension), however they differ with respect to their morphology with varying interpenetration levels (DATASET2: higher interpenetration, DATASET3: lower interpenetration). For all morphologies, 100 or more trajectory pairs were generated as a result of KMC simulations where a hole-electron pair separates and diffuses to the electrodes (topbottom boundary plane) aligned perpendicular to the Z-axis. All 


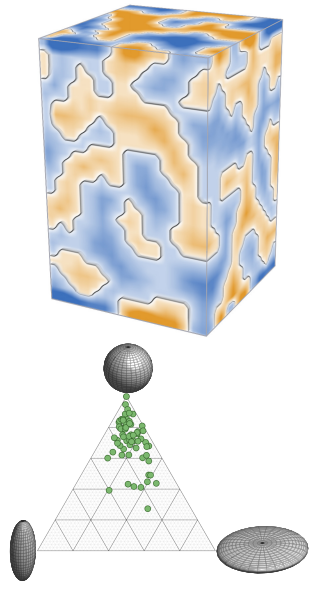

(a)

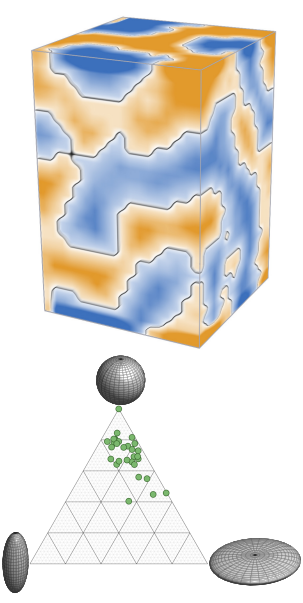

(b)
Figure 11: Morphology analysis. Distance fields of the morphology (top) and pocket shape distributions (bottom) of DATASET2 (a) and DATASET3 (b).

hole-electron pairs start at the same interface point between acceptor and donor material, hence they always start from the same pocket.

\subsection{Use case 1 - Comparison of two datasets}

In this use case, we compare DATASET2 and DATASET3, which present different levels of interpenetration.

First data inspection. The exploration starts with a first inspection of the datasets to verify the correctness of the data. Figure 10 shows both raw datasets as trajectories embedded in their morphology without periodicity and with expanded trajectories considering the periodic boundary conditions. The trajectory paths are clamped once they reach the electrodes. We can see that the morphologies are almost completely filled with trajectories. This has been expected and is a first test to verify the simulation and the model parameters. From the expanded visualization we can conclude that the trajectories for the morphology with less interpenetration (DATASET3) are spreading further out and are not as confined as in the other case. This information is very relevant for the domain scientist to identify the level of interpenetration necessary for a full diffusion of the charges in the morphology. However, it becomes obvious that neither the compact representation nor the periodic expansion diminishes the visual clutter, and hence are not appropriate for a detailed analysis of the data.

Morphology analysis. In this step, we take a closer look at the two morphologies. The visualization of the signed distance field of the morphologies shows different levels of interpenetration (Figure 11, top). The segmentation results in varying pocket counts and pocket sizes for respective datasets. Figure 11, bottom shows the distribution of the pocket shapes, which confirms that the DATASET3 features more planar structures. Note that due to the periodic boundary conditions, pockets can be leaving the volume on one boundary and
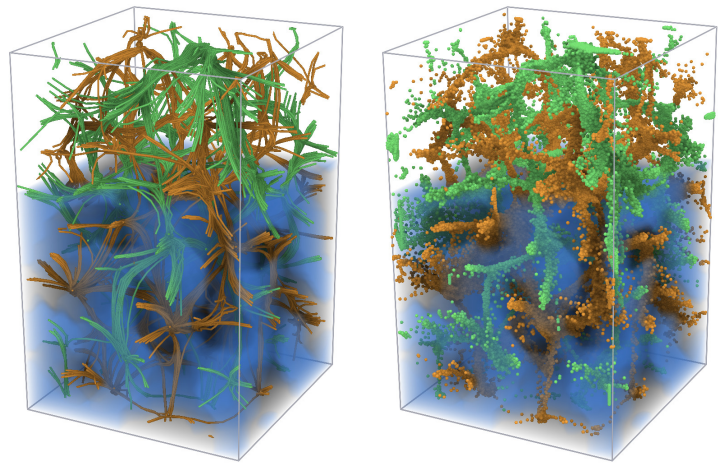

(a) DATASET2

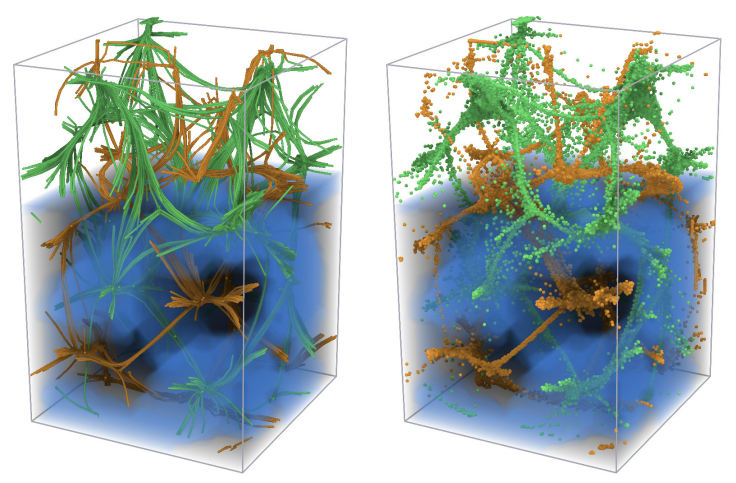

(b) DATASET3

Figure 12: Trajectory bundling. DATASET3 (bottom) exhibits more planar structures than DATASET2 (top). Charge positions shown on the right represent an intermediate state of the bundling process.

entering on the opposite side again. This continuity is reflected in the segmentation results.

Trajectory bundling. Looking at the raw trajectories gives not much information about the macroscopic charge transport. The high level movement of charges across the pockets is captured by the trajectory bundling relying on a spring mass system, see Figure 12. These visualizations show how the trajectories pass through the pockets when moving toward the electrodes. The bundling process can be interrupted and an intermediate state can be inspected at any time. It appears that the charges have no preferred way to pass through the pockets and the bundles enter and leave through all possible interfaces. However, observing the bundling process in the pockets already give interesting information about the charge distributions and the dwell time of charges in a pocket. In comparison, the two datasets reveal clearly that DATASET3 exhibits more planar transport structures than DATASET2. With a planar distribution, a charge interaction and recombination will be much less likely due to linear structures, and thus results in a longer convergence time (see Table 1). The bundling process allows to extract the mean path of the many Kinetic Monte Carlo trajectories that is more relevant for the efficiency than the detailed stochastic charge movement. However, no clear inference can be made from these 
images. Hence further exploration of the trajectories and pockets in the next stage is required.

Full abstraction. A more quantitative analysis is possible with a full abstraction of the charge flow. The full three dimensional embedding of the charge flow network (DATASET2) is depicted in Figure 13a. While the embedding reveals the full complexity of the transport process, the chord diagram provides a direct overview of the number and size of pockets as well as their valency (Figure 13c). Linking these two representations results in an intuitive interface for the exploration of the charge transport. The blue chords on the left side of the diagrams represent the pockets at the top electrode. The pockets of the bottom electrode correspond to the blue are in the top part of the chord diagrams. This already reveals an interesting facet of the dataset: multiple charge pairs are not separating and thus head toward the same electrode. In Figure $13 \mathrm{~b}$, the trajectories of one such a charge pair are depicted.

\subsection{Use case 2 - Pocket and trajectory exploration}

In this use case, we use the DATASET 1 to explore selected pockets and trajectories in more detail. The dataset is different from the use case 1 in terms of interpenetration and has a more complex morphology. The entire network is captured by the aggregated adjacency matrix and is represented using chord diagram in Figure 16a. Pockets can be selected in the chord diagram, in the 3D embedding, or via filtering using associated characteristic measures. With the selection of a pocket, one can also highlight all trajectories passing through pockets. We use two modes of pocket exploration using abstract representations: within the abstract visualizations and in the spatial embedding.

Case 2a. The first goal is to specify pockets with high a number of connections to other pockets. For this purpose the summary of all the trajectories in the chord diagram are very suitable Figure 16a. In this diagram we select pockets 1091, 1174 (donor) and 202, 196 (acceptor) showing the highest number of connections. Filtering for a trajectory that passes through all four pockets results in a trajectory pair shown in Figure 16b. Pockets 1091 and 202 are the starting nodes (Figure 16d). Pocket 1174 shows a high activity both for a single trajectory and the entire network. Interestingly, both pockets 1174 and 196 are immediate neighbors to the start pocket but do not receive much contribution from there. This can be an indicator for strong charge interaction moving the charges back toward their start position. In a second step we try to identify possible bottlenecks, therefore we focus on pockets with a low valency and select two pockets with valency 2 (Figure 14). The assumption is that low valency is a hint for a transport bottleneck. Inspecting the spatial embedding shows that both pockets act as connectors between two larger pockets with a very thigh connection. In this case this means that there is a strong correlation between the morphological and the transport bottleneck. This identification of specific pockets such as bottleneck is one of the goal for the domain scientist, and this can be achieved with the chord diagram.

Case 2b. In this case, we select a trajectory pair from the spatial network. This trajectory pair augmented with traversed pockets is depicted in Figure 15a where color encodes time. Two different

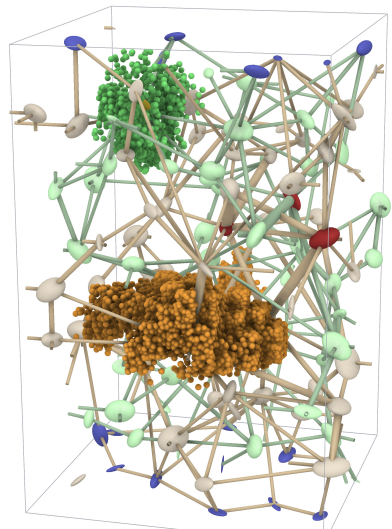

(a)

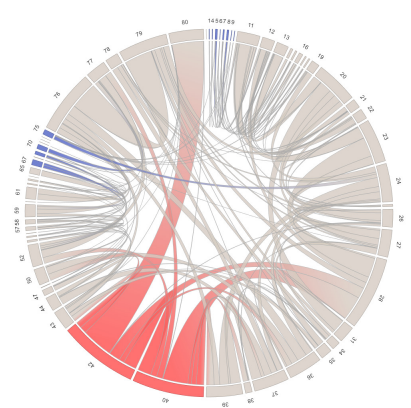

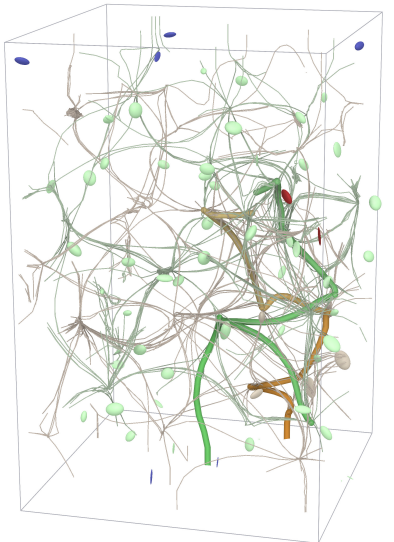

(b)

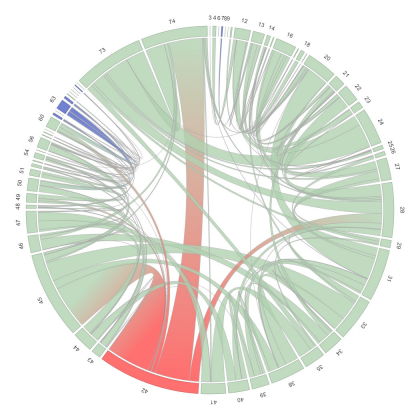

Figure 13: Charge flow network of DATASET2. (a) spatial network embedding with two highlighted pockets; associated charge distributions in green (electrons) and orange (holes). Pockets are represented by ellipsoids whose shape corresponds to the local charge distributions (red: start pockets; blue: end pockets at the electrodes; light orange/green: transit pockets). Periodic boundary conditions are indicated by open-ended connections. (b) trajectory inspection of a single charge pair where both electron and hole move toward the same electrode. (c) In depth inspection of the transport behavior is facilitated in combination with chord diagrams (hole left, electrons right). Both diagrams feature end pockets (blue) at the top and left which correspond to the bottom electrode and the top electrode, respectively. This indicates that charge pairs are not separating, as e.g. depicted in (b).

color scales are used for electron and hole, respectively. The large yellow region in the center is a hint of a large dwell time of the charges close to the exciton generation. This can be explained by electrostatic forces between the two charges but may have a negative effect on the transport efficiency bearing a high risk of losing the charge pair due to recombination. The second representation Figure $15 \mathrm{~b}$ shows the same pockets now colored with respect to the overall charge count, which is relatively high for relevant pockets. This shows that this behavior seems to be a general trend, which is not only present for the selected trajectory. In such scenarios, the spatial skeleton is useful for picking and selection of morphology 
Table 1: Computation time and data complexity involved in various stages of the charge flow network pipeline. The scaling is mostly depending on the number of charge positions, i.e. the length of each trajectory and, of course, the total number of trajectories. The size of the morphology, i.e. the volume, will only affect the segmentation and maxima extraction. Thus, trajectory bundling is most demanding since it involves computing the distance field and force field gradient as well as the relaxation for all trajectories until convergence.

\begin{tabular}{ccccccccc}
\hline Data & \multicolumn{3}{c}{ Morphology } & \multicolumn{2}{c}{ Trajectory } & Abstraction \\
\hline & Dimensions & Maxima Extraction [ms] & Segmentation [ms] & \# Trajectories & Total Charge Count & Bundling [ms] & Adjacency Matrix & Compute Adjacency [ms] \\
\hline DATASET 1 & $50 \times 50 \times 50$ & 960 & 255 & 200 & 1000000 & 19400 & $127 \times 127$ \\
DATASET2 & $18 \times 18 \times 28$ & 570 & 103 & 600 & 1800000 & 50330 & $72 \times 72$ \\
DATASET3 & $18 \times 18 \times 28$ & 573 & 102 & 600 & 4000000 & 72400 & $37 \times 37$ & 211 \\
\hline
\end{tabular}

pockets. The interaction during exploration is a key feature, which is facilitated in these abstract representations.

The next step is the exploration of the charge distribution in the respective pockets. Interesting properties are the distribution of charges within the pocket and during the transitions to its neighbors shown as histogram respective heat map on the interfaces Figure 9. Understanding the charge distribution within the pocket is a precursor for detecting the interrupted flow in a pocket.
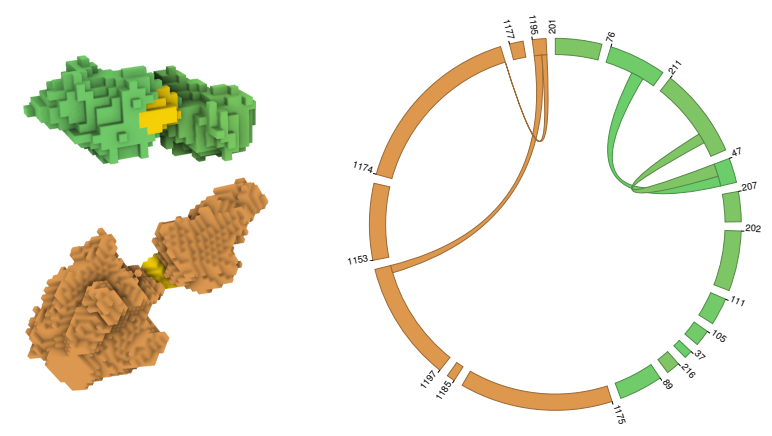

Figure 14: Exploration of pockets with two neighbors. Three segmented pockets of acceptor and donor channels form a narrow channel (left). These pockets are identified using the chord diagram of a single trajectory (right).

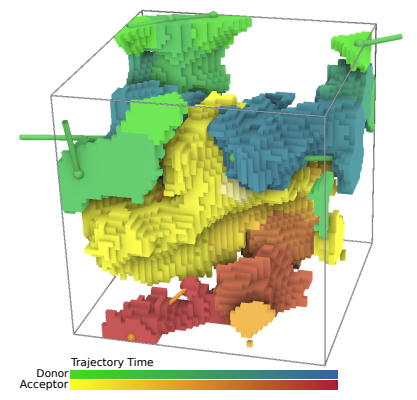

(a)

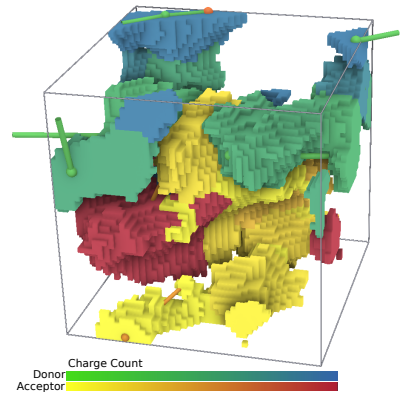

(b)
Figure 15: Single Trajectory Analysis of an electron-hole charge pair. Pockets traversed by either electron or hole are color mapped to (a) pocket entry time and (b) charge count.

\section{Conclusion}

In summary, we propose a novel way to represent and analyze charge trajectories using a flow network augmented with derived statistical information. The framework is assembled of three major parts that provide the backbone for visualization, interaction, and aggregation of geometric measures: morphology segmentation, trajectory bundling, and trajectory abstraction in one matrix. Linking these elements together facilitates a large set of exploration possibilities. Through this visualization system, it is the first time that the physicists have full access to the simulation results. The interactivity and linkage of all levels plays an important role for the understanding of the data. Questions of the domain experts include debugging of the simulation results by comparing inflow and outflow of charges in the pockets, evaluating parameters like external field strength, and comparing the efficiency for different morphologies. Some of the anticipated properties have been confirmed during the exploration but there have also been novel aspects that were not considered before. For example the role of the shape of the morphology pockets and the different behavior of charges in planar and linear regions, which is not represented by the typically used morphology measures such as domain size and volume to area ratio. Due to this observation we plan to make these geometric characteristics more quantitative in future development. The framework is growing by adding novel functionalities as the exploration of the data goes on. For the construction and augmentation of the network, new concepts have been introduced, which have the potential to be used in other application areas as well. This includes bundling and abstraction of the trajectories, which can also be of interest for other dynamic data. The visual and interactive analysis provided here gives the domain expert a quick and intuitive tool to analyze the trajectories and establish morphology-efficiency relationship necessary for the design of more efficient materials. To extend the comparison possibilities of the framework, we consider integrate more shape analysis tools for the morphology as proposed by Aboulhassan et al. [ASB*17].

\section{Acknowledgments}

This work was supported through grants from the Excellence Center at Linköping and Lund in Information Technology (ELLIIT) and the Swedish e-Science Research Centre (SeRC). M. Linares thanks the Swedish National Infrastructure for Computing (SNIC) for providing computing resources. The presented concepts have been developed and evaluated in the Inviwo framework (www. inviwo.org). 


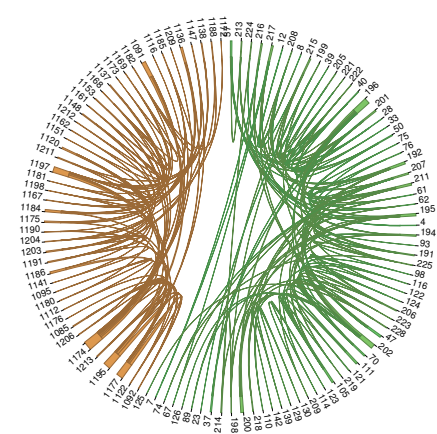

(a)

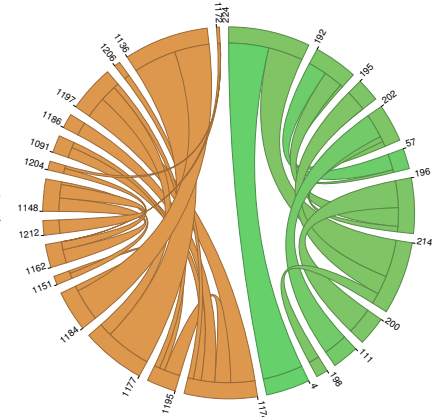

(b)

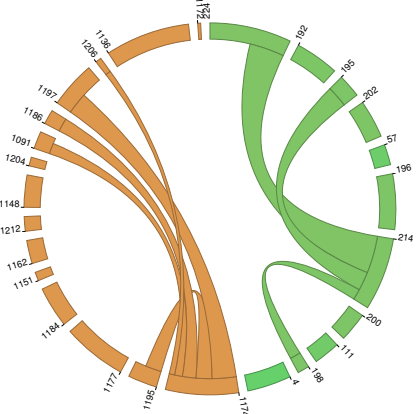

(c)

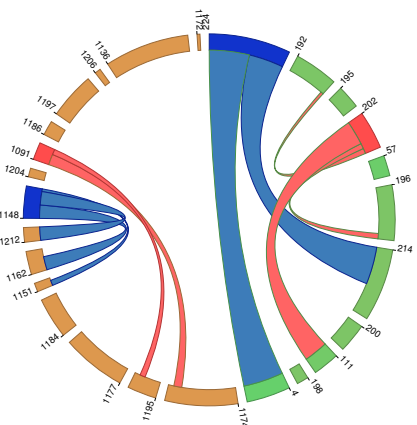

(d)

Figure 16: Chord diagram of the adjacency matrix derived from the charge flow network. The numbers relate to unique pocket IDs while colors differentiate electron and hole trajectories (green/orange), respectively. Red and blue pocket colors indicate start and end nodes of a trajectory. (a) aggregated transitions of all pockets. $(b)-(d)$ the trajectories of a single charge pair, i.e. an electron and a hole, are selected from the aggregated network. (c) connections of two pockets (IDs 214 and 1174), which exhibit higher activity within the trajectory. (d) connections of start and end nodes.

\section{References}

[AA12] ANDRIENKo N., ANDRIENKo G.: Visual analytics of movement: An overview of methods, tools and procedures. Information Visualization 12, 1 (2012), 3-24. doi : 10 .1177/1473871612457601.

[ABW*15] Aboulhassan A., Baum D., Wodo O., GanapaTHYSUBRAMANiAN B., AMASSIAN A., HADWiger M.: A novel framework for visual detection and exploration of performance bottlenecks in organic photovoltaic solar cell materials. In Computer Graphics Forum (2015). doi:10.1111/cgf.12652. 3

[AHK*17] Aldrich G., Hyman J., Karra S., Gable C., MakeDONSKA N., VisWANATHAN H., WOODRING J., HAMANN B.: Analysis and visualization of discrete fracture networks using a flow topology graph. IEEE Transactions on Visualization and Computer Graphics (2017). doi:10.1109/TVCG.2016.2582174.3

[ASB*17] Aboulhassan A., SiCAT R., Baum D., Wodo O., HaDWIGER M.: Comparative visual analysis of structure-performance relations in complex bulk-heterojunction morphologies. Computer Graphics Forum 36, 3 (2017), 329-339. doi : 10 .1111/cgf .13191. 3, 10

[BOH11] Bostock M., OgIEvetsky V., HeER J.: D ${ }^{3}$ data-driven documents. IEEE Transactions on Visualization and Computer Graphics 17, 12 (2011), 2301-2309. doi:10.1109/TVCG.2011.185. 7

[EH08] Edelsbrunner H., HARER J.: Persistent homology-a survey. Contemporary Mathematics 453 (2008), 257-282. 4

[EM90] Edelsbrunner H., MÜCKE P.: Simulation of simplicity: A technique to cope with degenerate cases in geometric algorithms. ACM Transactions on Graphics 9 (1990), 66-104. doi : $10.1145 / 77635$. 77639. 4

[GRWH12] GÜNTher D., ReIninghaus J., WAGner H., Hotz I.: Efficient computation of 3D Morse-Smale complexes and persistent homology using discrete morse theory. The Visual Computer 28, 10 (2012), 959-969. doi:10.1007/s00371-012-0726-8.3

[Hol06] HolTEN D.: Hierarchical edge bundles: Visualization of adjacency relations in hierarchical data. IEEE Transactions on Visualization and Computer Graphics 12, 5 (2006), 741-748. doi:10.1109/ TVCG. 2006.147 .3

[JLS12] Jakobsson M., Linares M., Stafström S.: Monte Carlo simulations of charge transport in organic systems with true off-diagonal disorder. The Journal of Chemical Physics 137 (2012), 114901. doi: $10.1063 / 1.4748796 .2$
[JSS*19] JÖNSSON D., STENETEG P., SUNDÉN E., ENGLUND R., Kottravel S., Falk M., Ynnerman A., Hotz I., Ropinski T.: Inviwo - a visualization system with usage abstraction levels. IEEE Transactions on Visualization and Computer Graphics (2019). To appear. 7

[KSB*09] Krzywinski M. I., Schein J. E., Birol I., Connors J., Gascoyne R., Horsman D., Jones S. J., Marra M. A.: Circos: An information aesthetic for comparative genomics. Genome Research 19,9 (2009). doi:10.1101/gr.092759.109.7

[KVL*17] Kottravel S., Volpi R., Linares M., Ropinski T., Hotz I.: Visual analysis of stochastic trajectory ensembles in organic solar cell design. Informatics. Special issue 'Scalable Interactive Visualization' 4, 3 (2017), 25. doi : 10 .3390/informatics 4030025. 2,3

[LHvdS01] Lindahl E., Hess B., VAN DeR Spoel D.: GROMACS 3.0: a package for molecular simulation and trajectory analysis. Molecular Modeling Annual 7, 8 (2001), 306-317. doi:10.1007/ s008940100. 3

[SPN*16] Shivashankar N., PranaV P., Natarajan V., VAN DE Weygaert R., Bos E. P., Rieder S.: Felix: A topology based framework for visual exploration of cosmic filaments. IEEE Transactions on Visualization and Computer Graphics 22, 6 (2016), 1745-1759. doi:10.1109/TVCG.2015.2452919.3

[ST94] SaITo T., ToriwaKi J. I.: New algorithms for Euclidean distance transformation of an n-dimensional digitized picture with applications. Pattern Recognition 27, 11 (1994), 1551-1565. doi : $10.1016 /$ $0031-3203$ (94) 90133-3. 4

[UMW*12] Ushizima D. M., Morozov D., Weber G. H., Bianchi A. G., Sethian J. A., Bethel E. W.: Augmented topological descriptors of pore networks for material science. IEEE Transaction on Visualization and Computer Graphics 18, 12 (2012), 2041-2050. doi:10.1109/TVCG.2012.200.3

[VKN*16] Volpi R., KotTravel S., Norby M. S., STAFSTRÖM S., LINARES M.: Effect of polarization on the mobility of C60: A kinetic monte carlo study. Journal of Chemical Theory and Comuputation 12, 2 (2016). doi:10.1021/acs.jctc.5b00975. 1,2,3

[VSL15] VOLPI R., STAFSTRÖM S., LINARES M.: Transition fields in organic materials: from percolation to inverted Marcus regime. a consistent Monte Carlo simulation in disordered PPV. The Journal of Chemical Physics 142 (2015), 094503. doi:10.1063/1.4913733.2 